\title{
The Quantum Poincaré-Birkhoff-Witt Theorem
}

\section{Roland Berger}

Département de Mathématiques, Faculté des Sciences et Techniques, 23, rue Docteur Paul Michelon, F-42023 Saint-Etienne Cedex 2, France*

Received July 20, 1990; in revised form February 22, 1991

Abstract. We define and study a wide class of associative algebras in which the Poincare-Birkhoff-Witt theorem is valid. This class includes numerous quantum algebras which recently appeared.

\section{Table of Contents}

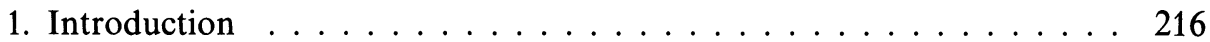

2. The $q$-PBW Theorem . . . . . . . . . . . . . . . . 217

2.1. The $q$-Algebras . . . . . . . . . . . . . . . . . . 217

2.2. The Invertible $q$-Algebras $\ldots \ldots \ldots \ldots . \ldots \ldots$

2.3. The Ordered Monomials of a $q$-Algebra . . . . . . . . . . . . 218

2.4. The $q$-Jacobi Sums . . . . . . . . . . . . . . . . . . . . 220

2.5. The $q$-Enveloping Algebras . . . . . . . . . . . . . . . . . . 220

2.6. Classical Examples . . . . . . . . . . . . . . . . . . 221

2.7. Non-Classical Examples . . . . . . . . . . . . . . . . 221

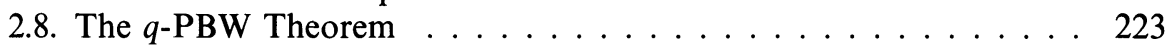

2.9. The Natural Filtration . . . . . . . . . . . . . . . 225

3. The QPBW-Theorem . . . . . . . . . . . . . . . . . . 227

3.1. The $h$-Adic Completion of a $q$-Enveloping Algebra . . . . . . . . . . 227

3.2. The Obstruction CPBW $\rightarrow$ QPBW . . . . . . . . . . . . . . 229

3.3. Quantization of Two Classical Properties . . . . . . . . . . . . 229

4. The QPBW-Theorem for $\mathbf{U}_{h}(s \ell(2)) \ldots \ldots \ldots . \ldots . \ldots . \ldots . \ldots 229$

4.1. Quantum Series in General . . . . . . . . . . . . . . . . . 229

4.2. The Algebra $\mathscr{U}_{h}(s \ell(2)) \ldots \ldots \ldots \ldots \ldots . \ldots \ldots$

4.3. Linearization of $\mathscr{U}_{h}(s \ell(2)) \ldots \ldots . \ldots . \ldots . \ldots . \ldots 230$

* This work was supported by the Laboratoire d'Analyse Fonctionnelle et Probabilités, Institut de Mathématiques et Informatique, 43, bd du 11 Novembre 1918, F-69622 Villeurbanne Cedex, France 
5. The Quantum Filtration . . . . . . . . . . . . . . . 232

5.1. The Planck Constant Has Degree $-1 \ldots \ldots \ldots \ldots 232$

5.2. Filtered Coproducts . . . . . . . . . . . . . . . . . 233

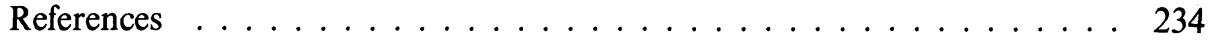

\section{Introduction}

According to Drinfel'd [6] a quantized enveloping algebra is a Hopf algebra over $\mathbb{C}[[h]]$ which is a deformation of a usual enveloping algebra $\mathscr{U}(\mathrm{g})$, with the Planck constant $h$ as a deformation parameter. For each simple Lie algebra g, Drinfeld and Jimbo have given a non-trivial deformation $\mathscr{U}_{h}(\mathrm{~g})$ of $\mathscr{U}(\mathrm{g})$, defined by generators and relations. A basic property of the usual enveloping algebras is the Poincaré-Birkhoff-Witt theorem (briefly CPBW theorem, C for classical). This theorem asserts that the ordered monomials in the generators of $\mathrm{g}$ form a basis of $\mathscr{U}(\mathrm{g})$. A natural expectation is that this theorem has an equivalent (called QPBW theorem, Q for quantum) after quantization. A QPBW theorem was stated by Jimbo [11] for $\mathscr{U}_{h}(s \ell(2))$, by Rosso [22] for $\mathscr{U}_{h}(s \ell(n))$ and by Lusztig [15] for the general Drinfeld-Jimbo algebra $\mathscr{U}_{h}(\mathrm{~g})$.

In this paper, we put the question of the PBW basis inside the general class of the associative algebras over $\mathbb{C}[[h]]$ defined by inhomogeneous quadratic relations. This class does not contain the algebras $\mathscr{U}_{h}(\mathfrak{g})$, but some subalgebras as the + part of the triangular decomposition of $\mathscr{U}_{h}(s \ell(n))$ belong to it. On the other hand, quadratic relations arise when one quantizes the algebra of the functions on the Lie group rather than the enveloping algebra (Manin [18]) and the existence of PBW basis also comes up in this case. Moreover, Priddy had already showed the interest of quadratic algebras with PBW basis for the construction of generalized Koszul resolutions [21].

A condition on the relations has to be required in order that the ordered monomials form a basis, even if the classical limit (obtained for $h \rightarrow 0$ ) has the same property. For example, consider the associative algebra $\mathbf{U}$ generated by $e_{1}, e_{2}, e_{3}$ with the following relations:

$$
\begin{aligned}
& e_{2} e_{1}=e_{1} e_{2}, \\
& e_{3} e_{1}=e_{1} e_{3}+h, \\
& e_{3} e_{2}=(1+h) e_{2} e_{3}
\end{aligned}
$$

The classical limit $\mathbf{U} / h \mathbf{U}$ is the polynomial algebra in $e_{1}, e_{2}, e_{3}$. But it is easy to see that $h$ divides zero in $\mathbf{U}$ (see Sect. 3.2) which makes the QPBW theorem impossible in this case.

The essential ingredient in the CPBW theorem is the Jacobi identity. In order to have a QPBW theorem, we propose a certain "Jacobi condition," satisfied by a lot of known examples, and which is a quantization of the Jacobi identity. Evidently, this condition avoids the pathology of the above example.

As a matter of fact, we begin to express our Jacobi condition in the context of the $q$-quantization (the $q$-analogues) initiated by Jimbo [11] and fully used since in relation with the so-called $q$-calculus [19]. For us, the $q$-quantization just consists in forgetting the parameter $h$. For example, if $q=1+h$, the above relation (1.3) 
becomes the $q$-commutation relation $e_{3} e_{2}=q e_{2} e_{3}$. So, we work first with algebras on any commutative ring $\mathbf{K}$. If we want to recover the $h$-quantization, we take $\mathbf{K}=\mathbb{C}[[h]]$.

Roughly speaking, our $q$-analogues of the enveloping algebras are defined as follows. Firstly, $q$-commutators $e_{j} e_{i}-q_{j i} e_{i} e_{j}$ play the role of the classical commutators $e_{j} e_{i}-e_{i} e_{j}$. Secondly, the $q$-commutators are (inhomogeneous) quadratic in the generators with a symmetry hypothesis on the quadratic part. For these $q$-algebras (see Sect. 2.1), we introduce the $q$-Jacobi sums $\mathbf{J}\left(e_{k}, e_{j}, e_{i}\right)$ which live in the tensor algebra. The Jacobi condition is fulfilled if each $\mathbf{J}\left(e_{k}, e_{j}, e_{i}\right)$ belongs to an explicit submodule $\mathscr{E}$ of the tensor algebra. A $q$-algebra with satisfies the Jacobi condition is called a q-enveloping algebra. We prove in Sect. 2.8 the PBW theorem for the $q$-enveloping algebras.

As in the classical case, the verification of the Jacobi condition is automatic. The following known algebras are $q$-enveloping algebras: the additive (multiplicative) $q$-analogue of the Weyl algebra due to Kuryshkin [14] (Jategaonkar [10]); the algebra of the infinitesimal shifts of the Woronowicz pseudo-group $\mathbf{S U}_{q}(2)$ [25]; the Manin algebra $\mathbf{M}_{q}(2)$ of the $2 \times 2$ quantum matrices $[17,18]$. To this list, we add a (probably unknown) $q$-Heisenberg algebra which we represent by three fundamental operators of the $q$-calculus. Other quantum algebras are deduced from $q$-enveloping algebras by reduction modulo an ideal or by localization. For example: The Manin algebras $\mathbf{G L}_{q}(2)$ and $\mathbf{S L}_{q}(2)$; the Podles quantum 2-sphere $[20,19]$; the Hayashi $q$-analogue of Weyl algebra [7].

In Part 3, we return to the $h$-quantization and we prove the expected QPBW theorem for the $h$-adic completion of any $q$-enveloping algebra. In Part 4 , we apply this result to $\mathscr{U}_{h}(s \ell(2))$.

A consequence of the CPBW theorem is that a finitely generated enveloping algebra on a field has no zero divisor $\neq 0$ and is Noetherian. We extend these two properties to the $q$-enveloping algebras and their completion. The extension is obtained by considering the $q$-enveloping algebras as iterated skew polynomial algebras and using well-known results of Non-Commutative Algebra [16].

The usual enveloping algebras are almost commutative for the natural filtration (i.e. the associated graded algebra is commutative). Unfortunately, this result does not hold after the $q$-quantization. However, at the $h$-level, we can restore the almost commutativity thanks to the following hypothesis: $h$ has degree -1 (Sect. 5.1). Roughly speaking, the quantum world appears as the negative part of a $\mathbb{Z}$-filtration. For the quantum Hopf algebras, the coproduct is generally filtered for the new filtration. Then, we can associate to the quantum algebra a classical object which is a graded Hopf-Poisson algebra (in the Drinfeld sense).

\section{The q-PBW Theorem}

2.1. The $q$-Algebras. In this paper, $\mathbf{K}$ is a commutative ring and $\mathbf{V}$ is a $\mathbf{K}$-module with a basis $\left(e_{1}, \ldots, e_{p}\right)$. The indexing set $\mathbf{I}=\{1, \ldots, p\}$ is endowed with its natural total ordering. For each pair $(i, j)$ of indices such that $1 \leqq i<j \leqq p$, we choose $q_{j i}$ in $\mathbf{K}$ and $\left\langle e_{j}, e_{i}\right\rangle$ in $\mathbf{K} \oplus \mathbf{V} \oplus \mathbf{V}^{\otimes 2}$. Then, we consider the associative $\mathbf{K}$-algebra $\mathbf{U}=\mathbf{U}_{K}(\mathbf{V}) / \mathbf{R}$, where $\mathbf{T}_{K}(\mathbf{V})$ is the tensor algebra of $\mathbf{V}$ and $\mathbf{R}$ is the two-sided ideal 
of $T_{K}(V)$ generated by the following elements:

$$
\mathbf{R}_{j i}=e_{j} \otimes e_{i}-q_{j i} e_{i} \otimes e_{j}-\left\langle e_{j}, e_{i}\right\rangle, \quad i<j .
$$

2.1.1. Definition. With the above notations, $\mathbf{U}$ is called a q-algebra if the following ordered quadratic symmetry holds:

(S) If, in the quadratic part $\sum_{k, \ell} \alpha_{j i}^{k \ell} e_{k} \otimes e_{\ell}$ of $\left\langle e_{j}, e_{i}\right\rangle$, the coefficient $\alpha_{j i}^{k \ell}$ does not vanish, then $i<k \leqq \ell<j$ and $k-i=j-\ell$.

The $q$-algebra $\mathbf{U}$ is called scalar (linear, affine, quadratic) if each $\left\langle e_{j}, e_{i}\right\rangle$ belongs to $\mathbf{K}\left(\mathbf{V}, \mathbf{K} \oplus \mathbf{V}, \mathbf{V}^{\otimes 2}\right)$. Obviously, in the affine case, the hypothesis (S) becomes superfluous. The $q$-algebra $\mathbf{U}$ is called classical if $q_{j i}=1$ for all $i<j$. In this case, if $\langle$,$\rangle is extended into an alternate bilinear application on \mathbf{V}^{\otimes 2}$, the two-sided ideal $\mathbf{R}$ is generated by the elements $\mathbf{R}_{u v}=u \otimes v-v \otimes u-\langle u, v\rangle, u \in \mathbf{V}, v \in \mathbf{V}$. But, apart from this particular case and the case studied in Sect. 2.2, $\langle u, v\rangle$ makes sense only if $u=e_{j}, v=e_{i}, i<j$.

2.2. The Invertible $q$-Algebras. The $q$-algebra $\mathbf{U}$ is called invertible if each $q_{j i}$ is invertible in $\mathbf{K}$. In this case, $\mathbf{R}$ can be generated by elements $\mathbf{R}_{i j}=e_{i} \otimes e_{j}-q_{i j} e_{j} \otimes e_{i}-$ $\left\langle e_{i}, e_{j}\right\rangle, i<j$, where $q_{i j}=q_{j i}^{-1}$ and the quadratic part of $\left\langle e_{i}, e_{j}\right\rangle$ has the property (S) for the inverse ordering (if $\mathbf{U}$ is affine, we have $\left.\left\langle e_{i}, e_{j}\right\rangle=-q_{i j}\left\langle e_{j}, e_{i}\right\rangle\right)$. So, $\mathbf{U}$ is also a $q$-algebra when $\mathbf{I}$ is endowed with the inverse total ordering. In general, $\mathbf{U}$ is not a $q$-algebra for any total ordering (because of (S)). Nevertheless, if $\mathbf{U}$ is affine and if $\mathbf{I}$ is endowed with another total ordering $\varliminf^{\prime}, \mathbf{U}$ is also a $q$-algebra for $\leqq$.

2.2.1. Example. Assume $q_{j i}=\left\langle e_{j}, e_{i}\right\rangle=0$ for all $i<j$. The $q$-algebra $\mathbf{U}$ is not invertible. Moreover, for any other total ordering, $\mathbf{U}$ is not a $q$-algebra.

2.3. The Ordered Monomials of a $q$-Algebra. The following result is the first step towards the $q$-PBW theorem. We denote by $\bar{a}$ the class in $\mathbf{U}$ of any element $a$ of $\mathrm{T}_{K}(\mathbf{V})$.

2.3.1. Theorem. Let $\mathbf{U}$ be a q-algebra. The unit 1 and the ordered monomials $\bar{e}_{i_{1}} \cdots \bar{e}_{l_{n}}$, $i_{1} \leqq \cdots \leqq i_{n}, n \geqq 1$, generate the $\mathbf{K}$-module $\mathbf{U}$.

In his proof of the CPBW theorem, Jacobson uses an index which measures the disorder of a non-commutative monomial $\bar{e}_{i_{1}} \otimes \cdots \otimes \bar{e}_{i_{n}}[8]$. Unfortunately, the presence of quadratic terms in $\left\langle e_{j}, e_{i}\right\rangle$ prevents this index playing its role. We start to introduce an alternative index.

The index Ind $(x)$ of a sequence $x=\left(i_{1}, \ldots, i_{n}\right)$ of elements of $\mathbf{I}$ is defined by the relations:

$$
\begin{aligned}
\operatorname{Ind}(x) & =\sum_{1 \leqq j<k \leqq n} \eta_{j k}, \\
\eta_{j k} & =0, \quad \text { if } \quad i_{j} \leqq i_{k}, \\
\eta_{j k} & =i_{j}-i_{k}, \quad \text { if } \quad i_{j}>i_{k} .
\end{aligned}
$$

(In the Jacobson index, 1 takes place of $i_{j}-i_{k}$ in (2.4)). It is clear that Ind $(\mathrm{x})=0$ if and only if $i_{1} \leqq \cdots \leqq i_{n}$. Once the following technical lemma is proved, we shall be able to fully use the ordered quadratic symmetry (S). 
2.3.2. Lemma. Let $x=\left(i_{1}, \ldots, i_{n}\right)$ be such that $i_{\ell}>i_{\ell+1}$ for a precise $\ell(1 \leqq \ell \leqq n-1)$. Let $r, s$ be two integers such that $i_{\ell+1} \leqq r \leqq s \leqq i_{\ell}$ and $r-i_{\ell+1}=i_{\ell}-s$. Then, for the sequence $y=\left(i_{1}, \ldots, i_{\ell-1}, r, s, i_{\ell+2}, \ldots, i_{n}\right)$, the inequality holds:

$$
\text { Ind }(y)<\operatorname{Ind}(x) \text {. }
$$

Proof. We have to compare the $\eta_{j k}$ of $x$ and the $\eta_{j k}^{\prime}$ of $y$. We distinguish four cases.

First Case. $\eta_{j k}=\eta_{j k}^{\prime}$ if $j$ and $k$ are different from $\ell$ and $\ell+1$.

Second Case. $\eta_{\ell \ell+1}=i_{\ell}-i_{\ell+1}>0$ and $\eta_{\ell \ell+1}^{\prime}=0$.

Third Case. If $1 \leqq j \leqq \ell-1$, we prove the inequality:

$$
\eta_{j \ell}+\eta_{j \ell+1} \geqq \eta_{j \ell}^{\prime}+\eta_{j \ell+1}^{\prime}
$$

For doing that, examine each of the following cases:
A. $i_{j} \leqq i_{\ell+1}$,
B. $i_{\ell+1}<i_{j} \leqq r$,
C. $r<i_{j} \leqq s$,
D. $s<i_{j} \leqq i_{\ell}$,
E. $i_{\ell}<i_{j}$.

In $\mathrm{A}, \eta_{j \ell+1}=\eta_{j \ell}^{\prime}=0$.

In $\mathrm{B}, \eta_{j \ell+1}=i_{j}-i_{\ell+1}$ and $\eta_{j \ell}^{\prime}=0$.

In $\mathrm{D}, \eta_{j \ell}=0$ and $\eta_{j \ell+1}^{\prime}=i_{j}-s$.

In $\mathrm{E}, \eta_{j \ell}=i_{j}-i_{\ell}$ and $\eta_{j \ell+1}^{\prime}=i_{j}-s$.

In $\mathrm{A}, \mathrm{B}$ or $\mathrm{C}, \eta_{j \ell}=\eta_{j \ell+1}^{\prime}=0$.

In $\mathrm{C}, \mathrm{D}$ or $\mathrm{E}, \eta_{j \ell+1}=i_{j}-i_{\ell+1}$ and $\eta_{j \ell}^{\prime}=i_{j}-r$.

Then, it is clear that (2.6) holds in A, B, C.

In $\mathrm{D}, \eta_{j \ell}+\eta_{j \ell+1}=i_{j}-i_{\ell+1}$,

$$
\begin{aligned}
\eta_{j \ell}^{\prime}+\eta_{j \ell+1}^{\prime} & =2 i_{j}-r-s, \\
& =2 i_{j}-i_{\ell}-i_{\ell+1} \quad \text { by hypothesis. }
\end{aligned}
$$

Hence, (2.6) holds in D.

In $\mathrm{E}, \eta_{j \ell}+\eta_{j \ell+1}=2 i_{j}-i_{\ell}-i_{\ell+1}$,

$$
\eta_{j \ell}^{\prime}+\eta_{j \ell+1}^{\prime}=2 i_{j}-r-s
$$

Hence, (2.6) holds in E.

Fourth Case. If $\ell+2 \leqq j \leqq n$, by examination of the same cases $\mathrm{A}$ to $\mathrm{E}$, we obtain the inequality:

$$
\eta_{\ell j}+\eta_{\ell+1 j} \geqq \eta_{\ell j}^{\prime}+\eta_{\ell+1 j}^{\prime}
$$

We leave the verification to the reader.

Putting together the four cases, we deduce (2.5).

2.2.3. Remark. The condition $r-i_{\ell+1}=i_{\ell}-s$ is essential. For example, the sequences $(4,4,4,4,1)$ and $(4,4,4,2,2)$ have the same index.

Proof of Theorem 2.3.1. We have to show that any (non-ordered) monomial $e_{i_{1}} \otimes \cdots \otimes e_{i_{n}}$ in $\mathbf{T}_{\boldsymbol{K}}(\mathbf{V})$ is congruent modulo $\mathbf{R}$ to an ordered monomial. Reasoning by induction, we assume the assertion for monomials of lower degree than $n$ and for those of degree $n$ which are of lower index than the index of $\left(i_{1}, \ldots, i_{n}\right)$. Let $\alpha \geqq 1$ be the index of $\left(i_{1}, \ldots, i_{n}\right)$ and let $\ell$ be such that $i_{\ell}>i_{\ell+1}$. The monomial $e_{i_{1}} \otimes \cdots \otimes e_{i_{n}}$ is congruent $\bmod R$ to the following expression:

$$
\begin{gathered}
q_{i_{i},+1} e_{i_{1}} \otimes \cdots \otimes e_{i_{\ell+1}} \otimes e_{i_{\ell}} \otimes \cdots \otimes e_{i_{n}} \\
\quad+e_{i_{1}} \otimes \cdots \otimes\left\langle e_{i}, e_{i_{\ell+1}}\right\rangle \otimes \cdots \otimes e_{i_{n}} .
\end{gathered}
$$


By the lemma, the first term has an index $<\alpha$. By the lemma and the hypothesis (S), the homogeneous part of degree $n$ of the second term has also an index $<\alpha$.

2.4. The $q$-Jacobi Sums. Let $\mathbf{U}$ be a $q$-algebra. For $p \geqq k>j>i \geqq 1$, the $q$-Jacobi $\operatorname{sum} \mathbf{J}\left(e_{k}, e_{j}, e_{i}\right)$ is the following element of $\mathbf{V} \oplus \mathbf{V}^{\otimes 2} \oplus \mathbf{V}^{\otimes 3}$ :

$$
\begin{aligned}
\mathrm{J}\left(e_{k}, e_{j}, e_{i}\right)= & \left\langle e_{k}, e_{j}\right\rangle \otimes e_{i}-q_{k i} q_{j i} e_{i} \otimes\left\langle e_{k}, e_{j}\right\rangle \\
& -q_{j i}\left\langle e_{k}, e_{i}\right\rangle \otimes e_{j}+q_{k j} e_{j} \otimes\left\langle e_{k}, e_{i}\right\rangle \\
& +q_{k j} q_{k i}\left\langle e_{j}, e_{i}\right\rangle \otimes e_{k}-e_{k} \otimes\left\langle e_{j}, e_{i}\right\rangle .
\end{aligned}
$$

2.4.1. Proposition. The q-Jacobi sums $\mathbf{J}\left(e_{k}, e_{j}, e_{i}\right)$ belong to the ideal $\mathbf{R}$. In particular, the following relations hold in $\mathbf{U}$ :

$$
\overline{\mathbf{J}\left(e_{k}, e_{j}, e_{i}\right)}=0, \quad k>j>i .
$$

(Recall that $\bar{a}$ denotes the class in $\mathbf{U}$ of $a \in \mathbf{T}_{K}(\mathbf{V})$ ).

Proof. Using the expression (2.1) of $\mathbf{R}_{j i}$, we have:

$$
\mathbf{J}\left(e_{k}, e_{j}, e_{i}\right)=\mathbf{J}^{\prime}+\mathbf{J}^{\prime \prime}
$$

where $\mathbf{J}^{\prime}\left(\mathbf{J}^{\prime \prime}\right)$ is the expression obtained from the second member of (2.9) by replacing $\left\langle e_{m}, e_{\ell}\right\rangle$ by $-\mathbf{R}_{m \ell}$ (respectively by $\left.e_{m} \otimes e_{\ell}-q_{m \ell} e_{\ell} \otimes e_{m}\right)$ for $(m, \ell)=(k, j),(k, i)$ and $(j, i)$. The reader will check by an easy computation the surprising fact that $\mathbf{J}^{\prime \prime}=0$.

It is interesting to notice that the hypothesis (S) does not occur in this proof. Moreover, in the classical case $q_{j i}=1$, the relation (2.10) is exactly the usual Jacobi identity in $\mathbf{U}$ endowed with the commutator $a b-b a$.

2.5. The q-Enveloping Algebras. The statement of the Jacobi condition requires the introduction of two submodules of $\mathbf{T}_{K}(\mathbf{V})$. The submodule $\mathscr{E}_{1}$ is generated by the elements $\mathbf{R}_{j i}, i<j$, and the submodule $\mathscr{E}_{2}$ is generated by the elements $e_{i} \otimes \mathbf{R}_{j i}$, $\mathbf{R}_{j i} \otimes e_{i}, e_{j} \otimes \mathbf{R}_{j i}, \mathbf{R}_{j i} \otimes e_{j}, i<j$. It is easy to prove the following lemma (using (S) for (ii)).

\subsubsection{Lemma.}

(i) $\mathscr{E}_{1} \cap(\mathbf{K} \oplus \mathbf{V})=\{0\}$,

(ii) $\mathscr{E}_{2} \cap\left(\mathbf{K} \oplus \mathbf{V} \oplus \mathbf{V}^{\otimes 2}\right)=\{0\}$,

(iii) $\mathscr{E}_{1} \cap \mathscr{E}_{2}=\{0\}$.

2.5.2. Definition. $A$ q-algebra $\mathbf{U}$ is called enveloping if $\mathbf{U}$ satisfies the following Jacobi condition: for all integers $i, j, k$ with $1 \leqq i<j<k \leqq p$, the $q$-Jacobi sum $\mathbf{J}\left(e_{k}, e_{j}, e_{i}\right)$ belongs to $\mathscr{E}=\mathscr{E}_{1} \oplus \mathscr{E}_{2}$.

So, the Jacobi condition strengthens the belonging to the ideal $\mathbf{R}$ expressed by Proposition 2.4.1.

Suppose that the $q$-algebra $\mathbf{U}$ is invertible. According to Sect. $2.2, \mathbf{J}\left(e_{k}, e_{j}, e_{i}\right)$ makes sense for any distinct integers $i, j, k$ in $\mathbf{I}$. Moreover, some relations hold between these $q$-Jacobi sums if the indices are permuted. For example, if $\mathbf{U}$ is affine, we have:

$$
\mathbf{J}\left(e_{j}, e_{k}, e_{i}\right)=-q_{j k} \mathbf{J}\left(e_{k}, e_{j}, e_{i}\right)
$$


These relations show that if $\mathbf{U}$ is affine and is enveloping for the natural ordering of $\mathbf{I}, \mathbf{U}$ is enveloping for any total ordering.

2.6. Classical Examples. At this stage, it is necessary to prove that the $q$-enveloping algebras contain the usual enveloping algebras. In fact, we prove a little more.

Let $\mathbf{U}$ be a $q$-algebra which is classical and affine. In this case (see Sect. 2.1), the ideal $\mathbf{R}$ and the module $\mathscr{E}_{1}$ are generated by the elements $\mathbf{R}_{u v}=u \otimes v-v \otimes u-$ $\langle u, v\rangle, u \in \mathbf{V}, v \in \mathbf{V}$. Write the decomposition of $\langle u, v\rangle$ in $\mathbf{V} \oplus \mathbf{K}$ by:

$$
\langle u, v\rangle=[u, v]+\varphi(u, v)
$$

Then, for $u=e_{k}, v=e_{j}, w=e_{i}, k>j>i$, we can decompose $\mathbf{J}(u, v, w)$ in the following manner:

$$
\mathbf{J}(u, v, w)=\sum^{\prime} \mathbf{R}_{[u, v] w}+\sum^{\prime}[[u, v] w]+\sum^{\prime} \varphi([u, v] w),
$$

where $\sum^{\prime}$ denotes the sum on the cyclic permutations. The second $\sum^{\prime}$ (the third $\sum^{\prime}$ ) belongs to $\mathbf{V}(\mathbf{K})$, while the first $\sum^{\prime}$ belongs to $\mathscr{E}_{1}$. Hence, according to Lemma 2.5.1, $\mathbf{J}(u, v, w)$ belongs to $\mathscr{E}$ if and only if the second and the third $\sum^{\prime}$ vanish. We have obtained:

2.6.1. Theorem. Let $\mathbf{U}$ be a classical, affine q-algebra. Let [,] (respectively $\varphi$ ) be the alternate $\mathbf{K}$-bilinear application from $\mathbf{V}^{\otimes 2}$ to $\mathbf{V}$ (respectively $\mathbf{K}$ ) defined by (2.13). Then, $\mathbf{U}$ is enveloping if and only if [,] makes $\mathbf{V}$ into a Lie algebra $\mathrm{g}$ and $\varphi$ is $a$ 2 -cocycle for the cohomology of $\mathrm{g}$. In this case, $\mathbf{U}$ is the Sridharan enveloping algebra [23] of the pair $(\mathfrak{g}, \varphi)$.

I am endebted to R. Ouzilou to have pointed out to me Sridharan's work. The two extreme cases of the Sridharan algebras correspond to:

(1) the pairs $(\mathfrak{g}, 0)$ which give the usual enveloping algebras,

(2) the pairs $(\mathfrak{g}, \varphi), \mathfrak{g}$ abelian and $\varphi$ alternate.

This latter case contains the Weyl algebras.

2.7. Non-Classical Examples. The verifications of the Jacobi condition will be left to the reader. We start by some scalar examples. Let $\mathbf{U}$ be a scalar $q$-algebra. Lemma 2.5.1 shows that $\mathbf{U}$ is enveloping if and only if each $\mathbf{J}\left(e_{k}, e_{j}, e_{i}\right)$ vanishes, i.e. if we have the relations:

$$
\left(1-q_{k i} q_{j i}\right)\left\langle e_{k}, e_{j}\right\rangle=\left(q_{k j}-q_{j i}\right)\left\langle e_{k}, e_{i}\right\rangle=\left(q_{k i} q_{k j}-1\right)\left\langle e_{j}, e_{i}\right\rangle=0 .
$$

2.7.1. Example. A $q$-algebra such that each $\left\langle e_{j}, e_{i}\right\rangle$ vanishes is called pure. Any pure q-algebra is enveloping. It is the case of the Jategaonkar multiplicative analogue of the Weyl algebra [10], generated by $e_{1}, \ldots, e_{n}, e_{1}^{*}, \ldots, e_{n}^{*}$, with the relations:

$$
\begin{aligned}
e_{i} e_{j}-e_{j} e_{i} & =e_{i}^{*} e_{j}^{*}-e_{j}^{*} e_{i}^{*}=e_{i} e_{j}^{*}-e_{j}^{*} e_{i}=0, \quad i \neq j, \\
e_{i} e_{i}^{*} & =q_{i} e_{i}^{*} e_{i},
\end{aligned}
$$

where $q_{1}, \ldots, q_{n}$ are given in $\mathbf{K}$. The case $n=1$ is called a quantum plane by Manin $[17,18]$.

2.7.2 Example. With the same notations, the additive analogue of the Weyl algebra, defined by the relations $(2.15)$ and the following:

$$
e_{i} e_{i}^{*}-q_{i} e_{i}^{*} e_{i}=1,
$$


is also a $q$-enveloping algebra. This algebra was introduced in Quantum Physics by Kuryshkin [14] and studied by Jannussis-Brodimas-Sourlas [9].

The next linear examples is new in our opinion.

2.7.3. Example. Let $q$ be a real $>0, q \neq 1$. We denote by $f$ a function of a real variable $x$. The functional space which contains $f$ is not specified. We consider the operators $\delta_{q}, \tau_{q}, \hat{x}$ acting on $f$ by the formulas:

$$
\begin{aligned}
\delta_{q}(f)(x) & =\frac{f(x)-f(q x)}{(1-q) x}, \\
\tau_{q}(f)(x) & =f(q x), \\
\hat{x}(f)(x) & =x f(x) .
\end{aligned}
$$

In $q$-calculus, $\delta_{q}$ is the $q$-derivation and $\tau_{q}$ is the $q$-shift (see e.g. Wallisser [24]). It is straightforward to verify the following q-analogues of the Heisenberg commutation relations:

$$
\begin{aligned}
\delta_{q} \hat{x}-\hat{x} \delta_{q} & =\tau_{q}, \\
\delta_{q} \tau_{q} & =q \tau_{q} \delta_{q}, \\
\tau_{q} \hat{x} & =q \hat{x} \tau_{q} .
\end{aligned}
$$

The relations (2.21)-(2.23) define a $q$-enveloping algebra. We call it the $q$-Heisenberg algebra.

2.7.4. Example. The algebra generated by $e_{1}, e_{2}, e_{3}$ with the relations

$$
\begin{aligned}
e_{2} e_{1} & =q e_{1} e_{2}, \\
e_{3} e_{i}-q^{\prime} e_{i} e_{3} & =\alpha e_{i}, \quad i=1,2,
\end{aligned}
$$

is a $q$-enveloping algebra. Actually, we have:

$$
\mathbf{J}\left(e_{3}, e_{2}, e_{1}\right)=\alpha\left(1+q^{\prime}\right)\left(e_{2} \otimes e_{1}-q e_{1} \otimes e_{2}\right) .
$$

2.7.5. Example. The algebra generated by $e_{1}, e_{2}, e_{3}$ with the relations

$$
\begin{aligned}
& e_{2} e_{1}-q e_{1} e_{2}=\alpha e_{1}, \\
& e_{3} e_{2}-q e_{2} e_{3}=\alpha e_{3}, \\
& e_{3} e_{1}-q^{\prime} e_{1} e_{3}=\alpha^{\prime} e_{2}+\alpha^{\prime \prime} e_{2}^{2},
\end{aligned}
$$

is a $q$-enveloping algebra for the ordering $e_{1}<e_{2}<e_{3}$. An example of such an algebra for $\alpha^{\prime \prime}=0$ is provided by the infinitesimal shifts of Woronowicz ([25], Table 7). An example for $\alpha^{\prime \prime} \neq 0$ is provided by three of the four relations defining the quantum sphere $S_{q}^{2}$ of Podles $[20,19]$.

2.7.6. Example. The Manin algebra $\mathbf{M}_{q}(2, \mathbf{K})$ of the $2 \times 2$ quantum matrices $[17,18]$ is generated by $a, b, c, d$ with the relations:

$$
b a=q a b, \quad c a=q a c, \quad d c=q c d, \quad d b=q b d, \quad c b=b c, \quad d a-a d=\left(q-q^{-1}\right) b c .
$$


It is a $q$-algebra for the ordering $a<b<c<d$. We find:

$$
\begin{aligned}
& \mathbf{J}(d, b, a)=\left(1-q^{2}\right) b \otimes(c \otimes b-b \otimes c), \\
& \mathbf{J}(d, c, a)=\left(q^{2}-1\right)(c \otimes b-b \otimes c) \otimes c .
\end{aligned}
$$

Hence, this $q$-algebra is enveloping. A consequence of our $q$-PBW theorem (Sect. 2.8) is that the monomials $a^{i} b^{j} c^{k} d^{\ell}$ realize a basis of $\mathbf{M}_{q}(2, \mathbf{K})$.

2.7.7. Example. Hayashi has introduced a more sophisticated version of the $q$-Weyl algebra than the above one (Example 2.7.2). His purpose is to get bosonic representations for the types $\mathbf{A}_{n}$ and $\mathbf{C}_{n}$ of the Drinfeld-Jimbo quantum algebras [7]. Let $\mathbf{U}$ be the algebra generated by $\omega_{1}, \ldots, \omega_{n}, \psi_{1}, \ldots, \psi_{n}, \psi_{1}^{*}, \ldots, \psi_{n}^{*}$, with the relations:

$$
\begin{gathered}
\psi_{j} \psi_{i}-\psi_{i} \psi_{j}=\psi_{j}^{*} \psi_{i}^{*}-\psi_{i}^{*} \psi_{j}^{*}=\omega_{j} \omega_{i}-\omega_{i} \omega_{j}=0, \quad i<j, \\
\omega_{j} \psi_{i}-q^{-\delta_{i j}} \psi_{i} \omega_{j}=\psi_{j}^{*} \omega_{i}-q^{-\delta_{i j}} \omega_{i} \psi_{j}^{*}=0, \\
\psi_{j}^{*} \psi_{i}=\psi_{i} \psi_{j}^{*}, \quad i \neq j \\
\psi_{i}^{*} \psi_{i}-q^{2} \psi_{i} \psi_{i}^{*}=-q^{2} \omega_{i}^{2} .
\end{gathered}
$$

For the ordering $\psi_{1}<\cdots<\psi_{n}<\omega_{1}<\cdots<\omega_{n}<\psi_{1}^{*}<\cdots<\psi_{n}^{*}$, $\mathbf{U}$ is a $q$-enveloping algebra. To recover the Hayashi algebra $\mathscr{A}_{q}^{-}$, take $\mathbf{K}=\mathbb{C}$ and consider the multiplicative monoid $\mathbf{S}$ generated by $\omega_{1}, \ldots, \omega_{n}$. We shall see in Sect. 2.9 that any element of $\mathbf{S}$ is not a zero divisor. In addition, the relations (2.33) are pure. Hence, $\mathbf{S}$ is a regular Ore set and the localization $\mathbf{S}^{-1} \mathbf{U}$ of $\mathbf{U}$ at $\mathbf{S}$ exists (see [4] or [16] for the quotient rings $\mathbf{S}^{-1} \mathbf{A}$ with denominators in $\mathbf{S}$ ). Then $\mathscr{A}_{q}^{-}$is $\mathbf{S}^{-1} \mathbf{U}$ modulo the ideal generated by $\psi_{i} \psi_{i}^{*}-q^{2} \psi_{i}^{*} \psi_{i}-\omega_{i}^{-2}, i=1, \ldots, n$.

\subsection{The $q$-PBW Theorem}

2.8.1. Theorem. Let $\mathbf{U}$ be a q-enveloping algebra. The unit 1 and the ordered monomials $\overline{e_{i_{1}}} \cdots \overline{e_{i_{n}}}, i_{1} \leqq \cdots \leqq i_{n}, n \geqq 1$, form a basis of the $\mathbf{K}$-module $\mathbf{U}$.

As in Jacobson's proof of the CPBW theorem [8], it suffices to prove the existence of a certain map $\sigma$ from non-commutative polynomials to commutative polynomials.

2.8.2. Lemma. There is a $\mathbf{K}$-linear map $\sigma$ from $\mathbf{T}_{\boldsymbol{K}}(\mathbf{V})$ to $\mathbf{S}_{\boldsymbol{K}}(\mathbf{V})$ (the symmetric algebra of V) such that:

(i) $\sigma(1)=1$,

(ii) $\sigma\left(e_{i_{1}} \otimes \cdots \otimes e_{i_{n}}\right)=e_{i_{1}} \cdots e_{i_{n}}$ if $i_{1} \leqq \cdots \leqq i_{n}$,

(iii) if $i_{k}>i_{k+1}$ in the sequence $\left(i_{1}, \ldots, i_{n}\right)$, we have:

$$
\begin{aligned}
\sigma\left(e_{i_{1}} \otimes \cdots \otimes e_{i_{n}}\right)= & q_{i_{k} i_{k+1}} \sigma\left(e_{i_{1}} \otimes \cdots \otimes e_{i_{k+1}} \otimes e_{i_{k}} \otimes \cdots \otimes e_{i_{n}}\right) \\
& +\sigma\left(e_{i_{1}} \otimes \cdots \otimes\left\langle e_{i_{k}}, e_{i_{k+1}}\right\rangle \otimes \cdots \otimes e_{i_{n}}\right) .
\end{aligned}
$$

If we suppose this lemma, $\sigma$ goes through the quotient and defines a K-linear map $\bar{\sigma}: \mathbf{U} \rightarrow \mathbf{S}_{K}(\mathbf{V})$. Moreover, $\bar{\sigma}$ sends the family of the ordered monomials of $\mathbf{U}$ into the free family of the commutative monomials of $S_{K}(V)$. Hence, the source family is free, which is Theorem 2.8.1, taking Theorem 2.3.1 into account. Notice that $\bar{\sigma}^{-1}$ is a standard quantization correspondence in our sense [2]. But here, the enveloping algebra $\mathbf{U}$ is a lot more general. 
Proof of Lemma 2.8.2. Let $\mathbf{T}^{n}(\mathbf{V})\left(\mathbf{T}_{n}(\mathbf{V})\right), n \in \mathbb{N}$, be the submodules defining the natural grading (filtration) of $\mathbf{T}_{K}(\mathbf{V})$. For $\alpha \in \mathbb{N}, \mathbf{T}^{n}(\mathbf{V})_{\alpha}$ denotes the submodule of $\mathbf{T}^{n}(\mathbf{V})$ generated by the monomials of index $\leqq \alpha$ (see Sect. 2.3 for the index). Suppose that $\sigma$ is defined on $\mathbf{T}_{n-1}(\mathbf{V})$ with (i)-(iii). We define $\sigma$ on $\mathbf{T}^{n}(\mathbf{V})_{0}$ by (ii). Now, suppose that $\sigma$ is defined on $\mathbf{T}_{n-1}(\mathbf{V}) \otimes \mathbf{T}^{n}(\mathbf{V})_{\alpha-1}$. with (i)-(iii), and choose $e_{i_{1}} \otimes \cdots \otimes e_{i_{n}}$ with index $\alpha \geqq 1$. There is an integer $k$ such that $i_{k}>i_{k+1}$. We define $\sigma\left(e_{i_{1}} \otimes \cdots \otimes e_{i_{n}}\right)$ by (2.36). This makes sense since Lemma 2.3.2 and the symmetry (S) assure that the $n$-degree monomials on the right-hand side of (2.36) have an index $<\alpha$. Now, we have to verify that our definition does not depend on the choice of $\left(i_{k}, i_{k+1}\right)$ such that $i_{k}>i_{k+1}$. Let $\left(i_{\ell}, i_{\ell+1}\right)$ be another pair such that $i_{\ell}>i_{\ell+1}$. As in Jacobson, we distinguish the two cases: I. $\ell>k+1$, and II. $\ell=k+1$.

The Case $I$ is easy (it does not involve the Jacobi condition) and is discussed as in Jacobson. In the case II, set $u=e_{i_{k}}, v=e_{i_{k+1}}=e_{i,}, w=e_{i_{\prime+1}}, q_{u v}=q_{i_{k} i_{k+1}}$ and so on. The induction hypothesis allows to transform the right-hand side of (2.36) into:

$$
\begin{gathered}
q_{u v} q_{u w} q_{v w} \sigma(\cdots w \otimes v \otimes u \cdots)+q_{u v} q_{u w} \sigma(\cdots\langle v, w\rangle \otimes u \cdots) \\
+q_{u v} \sigma(\cdots v \otimes\langle u, w\rangle \cdots)+\sigma(\cdots\langle u, v\rangle \otimes w \cdots) .
\end{gathered}
$$

In a similar fashion, if we start from $q_{v w} \sigma(\cdots u \otimes w \otimes v \cdots)+\sigma(\cdots u \otimes\langle v, w\rangle \cdots)$, we obtain:

$$
\begin{aligned}
& q_{v w} q_{u w} q_{u v} \sigma(\cdots w \otimes v \otimes u \cdots)+q_{v w} q_{u w} \sigma(\cdots w \otimes\langle u, v\rangle \cdots) \\
& +q_{v w} \sigma(\cdots\langle u, w\rangle \otimes v \cdots)+\sigma(\cdots u \otimes\langle v, w\rangle \cdots) .
\end{aligned}
$$

The difference between (2.37) and (2.38) is written $\sigma(\mathbf{X})$, where $\mathbf{X}$ is expressed by means of a $q$-Jacobi sum:

$$
\mathbf{X}=e_{i_{1}} \otimes \cdots \otimes e_{i_{k+1}} \otimes \mathbf{J}(u, v, w) \otimes e_{i_{k+3}} \otimes \cdots \otimes e_{i_{n}} .
$$

As the q-algebra $\mathbf{U}$ is enveloping, we have $\mathbf{J}(u, v, w)=\mathbf{J}_{1}(u, v, w)+\mathbf{J}_{2}(u, v, w)$, where $\mathbf{J}_{i}(u, v, w) \in \mathscr{E}_{i}, i=1,2$. We write $\mathbf{X}=\mathbf{X}_{1}+\mathbf{X}_{2}$, where $\mathbf{X}_{i}$ is obtained by replacing $\mathbf{J}$ by $\mathbf{J}_{i}$ in (2.39).

For convenience, for $a \in \mathbf{T}(\mathbf{V})$, we set

$$
f(a)=e_{i_{1}} \otimes \cdots \otimes e_{i_{k-1}} \otimes a \otimes e_{i_{k+3}} \otimes \cdots \otimes e_{i_{n}} .
$$

The element $\mathbf{X}_{1}$ is a linear combination of the $f\left(\mathbf{R}_{j i}\right)$, each of them belonging to $\mathbf{T}_{n-1}(\mathbf{V})$. The induction works out and provides $\sigma\left(f\left(\mathbf{R}_{j i}\right)\right)=0$, hence $\sigma\left(\mathbf{X}_{1}\right)=0$.

To prove $\sigma\left(\mathbf{X}_{2}\right)=0$ is more delicate. We decompose 3-degree homogeneous part of $\mathbf{J}_{2}(u, v, w)$ in the following sum:

$$
\sum c_{r s t} e_{r} \otimes e_{s} \otimes e_{t}, \quad c_{r s t} \neq 0 .
$$

By construction, $f\left(e_{r} \otimes e_{s} \otimes e_{t}\right)$ has an index $<\alpha$. On the other hand, by definition of $\mathscr{E}_{2}, \mathbf{J}_{2}(u, v, w)$ equals to the following sum:

$$
\sum_{i<j}\left[d_{j i i} \mathbf{R}_{j i} \otimes e_{i}+d_{i j i} e_{i} \otimes \mathbf{R}_{j i}+d_{j i j} \mathbf{R}_{j i} \otimes e_{j}+d_{j j i} e_{j} \otimes \mathbf{R}_{j i}\right]
$$

It suffices to prove the assertion:

$$
\text { If } d_{r s t} \neq 0, \text { then } f\left(e_{r} \otimes e_{s} \otimes e_{t}\right) \text { has an index }<\alpha \text {. }
$$


Indeed, the induction would work out:

$$
\sigma\left(f\left(\mathbf{R}_{r s} \otimes e_{t} \text { or } e_{r} \otimes \mathbf{R}_{s t}\right)\right)=0, \text { hence } \sigma\left(\mathbf{X}_{2}\right)=0 .
$$

Proof of (2.42). Fix $i<j$ such that $j-i$ has the highest value in the sum (2.41). By comparison between (2.40) and (2.41), we obtain:

$$
\begin{gathered}
\sum_{r s t} c_{r s t} e_{r} \otimes e_{s} \otimes e_{t}=\mathbf{S}_{j i}+\mathbf{S}_{j i}^{\prime}+\mathbf{S}_{j i}^{\prime \prime}+\mathbf{S}^{\prime \prime \prime}, \\
\mathbf{S}_{j i}=d_{j i i} e_{j} \otimes e_{i} \otimes e_{i}+\left(d_{i j i}-q_{j i} d_{j i i}\right) e_{i} \otimes e_{j} \otimes e_{i}-q_{j i} d_{i j i} e_{i} \otimes e_{i} \otimes e_{j}, \\
\mathbf{S}_{j i}^{\prime}=d_{j j i} e_{j} \otimes e_{j} \otimes e_{i}+\left(d_{j i j}-q_{j i} d_{j j i}\right) e_{j} \otimes e_{i} \otimes e_{j}-q_{j i} d_{j i j} e_{i} \otimes e_{j} \otimes e_{j}, \\
\left.\mathbf{S}_{j i}^{\prime \prime}=\sum \mathbf{S}_{j^{\prime} i^{\prime}}+\mathbf{S}_{j^{\prime} i^{\prime}}^{\prime} \quad \text { (sum over } i^{\prime}<j^{\prime},\left(i^{\prime}, j^{\prime}\right) \neq(i, j)\right) .
\end{gathered}
$$

As $\mathbf{S}^{\prime \prime \prime}$ is deduced from the quadratic parts of the $\langle$,$\rangle , we find in it either monomials$ $e_{k} \otimes e_{\ell} \otimes e_{m}$ with distincts $k, \ell, m$, or monomials $e_{k} \otimes e_{k} \otimes e_{m}, e_{m} \otimes e_{k} \otimes e_{k}$ with $|k-m|<j-i$ (because of $(S))$. Hence, the monomials of $\mathbf{S}_{j i}$ are linearly independent from all the monomials of $\mathbf{S}_{j i}^{\prime}+\mathbf{S}_{j i}^{\prime \prime}+\mathbf{S}^{\prime \prime \prime}$. If $d_{j i i} \neq 0$, then $c_{j i i}=d_{j i i}$ and $f\left(e_{j} \otimes e_{i} \otimes e_{i}\right)$ has an index $<\alpha$ by construction. Now, if $d_{i j i} \neq 0$ and $d_{i j i} \neq q_{j i} d_{j i i}$, then $c_{i j i}=d_{i j i}-q_{j i} d_{j i i}$, and $f\left(e_{i} \otimes e_{j} \otimes e_{i}\right)$ has an index $<\alpha$. On the other hand, if $d_{i j i} \neq 0$ and $d_{i j i}=q_{j i} d_{j i i}$, then $d_{j i i} \neq 0$ and $f\left(e_{j} \otimes e_{i} \otimes e_{i}\right)$ has an index $<\alpha$. Lemma 2.3.2 shows that $f\left(e_{i} \otimes e_{j} \otimes e_{i}\right)$ has also an index $<\alpha$. We identically proceed for $d_{j i j}$ and $d_{j j i}$. Then, we have to repeat the same argument for a lower value of $j-i$. Finally, for the lowest value $j-i=1$, the argument is clear because $\mathbf{S}^{\prime \prime \prime}=0$ in this case.

2.8.3. Remark. The difficulty of the previous proof comes from the quadratic terms of $\left\langle e_{j}, e_{i}\right\rangle$. If $\mathbf{U}$ is affine, the proof essentially becomes the Jacobson one (his index suffices) and it works out again in the infinite-dimensional case.

An immediate consequence of Theorem 2.8.1 is that the canonical mapping $V \rightarrow U$ is injective. From now on, we identify $e_{i}$ to its class $\overline{e_{i}}$.

2.9. The Natural Filtration. Let $\mathbf{U}$ be a $q$-enveloping algebra. We denote by $Q\left(e_{j}, e_{i}\right)$ the quadratic part of $\left\langle e_{j}, e_{i}\right\rangle$. The algebra $\mathbf{Q}(\mathbf{U})=\mathbf{T}_{K}(\mathbf{V}) / \mathbf{R}^{\prime}, \mathbf{R}^{\prime}$ ideal generated by the elements $e_{j} \otimes e_{i}-q_{j i} e_{i} \otimes e_{j}-Q\left(e_{j}, e_{i}\right), i<j$, is called the quadratic algebra associated with $\mathbf{U}$. It is easy to see that $\mathbf{Q}(\mathbf{U})$ is also a q-enveloping algebra. If $\mathbf{U}$ is a Sridharan algebra (Sect. 2.6), $\mathbf{Q}(\mathbf{U})$ is the symmetric algebra $\mathbf{S}_{K}(\mathbf{V})$. In the general case, $\mathbf{Q}(\mathbf{U})$ plays the role of $\mathbf{S}_{K}(\mathbf{V})$. To see this, we introduce the natural filtration of $\mathbf{U}$.

For $m \in \mathbb{N}, \mathbf{U}^{m}$ is the submodule of $\mathbf{U}$ generated by the products of $k$ elements in $\mathbf{V}, k \leqq m$. Then, $\mathbf{U}$ is a $\mathbf{K}$-algebra filtered by the $\mathbf{U}^{m}$. Denote by $g r(\mathbf{U})$ its associated graded algebra. The grading of $g r(\mathbf{U})$ is formed by the $g r_{m}(\mathbf{U})=\mathbf{U}^{m} / \mathbf{U}^{m-1}, m \in \mathbb{N}$, and $\mathbf{V}=g r_{1}(\mathbf{U})$ generates the algebra $g r(\mathbf{U})$. The $q$-PBW theorem easily implies the following lemma:

2.9.1. Lemma. If $\times$ is the product of $\operatorname{gr}(\mathbf{U})$, the elements 1 and $e_{i_{1}} \times \cdots \times e_{i_{n}}$, $i_{1} \leqq \cdots \leqq i_{n}, n \geqq 1$, realize a basis of the $\mathbf{K}$-module $\operatorname{gr}(\mathbf{U})$.

With the notations of Sect. 2.1, the defining relations of $\mathbf{Q}(\mathbf{U})$ are:

$$
e_{j} e_{i}=q_{j i} e_{i} e_{j}+\sum_{k \ell} \alpha_{j i}^{k \ell} e_{k} e_{\ell}, \quad i<j .
$$


Now, in $\mathbf{U}^{2} / \mathbf{U}^{1}$, we have:

$$
e_{j} \times e_{i}=q_{j i} e_{i} \times e_{j}+\sum_{k \ell} \alpha_{j i}^{k \ell} e_{k} \times e_{\ell \cdot} \quad i<j .
$$

Hence, the canonical map $\mathbf{V} \rightarrow \operatorname{gr}(\mathbf{U})$ extends to a unique algebra morphism $\psi: \mathbf{Q}(\mathbf{U}) \rightarrow \operatorname{gr}(\mathbf{U})$. But the image by $\psi$ of the PBW basis of $\mathbf{Q}(\mathbf{U})$ is the basis of Lemma 2.9.1. So, we obtain the following generalization of a classical result [4].

2.9.2. Theorem. Let $\mathrm{U}$ be a q-enveloping algebra. The algebra morphism $\psi$ : $\mathbf{Q}(\mathbf{U}) \rightarrow \operatorname{gr}(\mathbf{U})$ extending the identity of $\mathbf{V}$ is an isomorphism.

Suppose $\mathbf{Q}(\mathbf{U})$ commutative. The equality (2.44) and the $q$-PBW theorem imply that $q_{j i}=1$ and $\alpha_{j i}^{k \ell}=0, i<j$. We can claim:

2.9.3. Corollary. Let $\mathrm{U}$ be a q-enveloping algebra. The associated graded algebra $\operatorname{gr}(\mathbf{U})$ is commutative if and only if $\mathbf{U}$ is a Sridharan enveloping algebra.

The latter results are rather disappointing. For example, if $\mathbf{U}$ is quadratic, $g r(\mathbf{U})$ is isomorphic to $\mathbf{U}$ ! In fact, the Planck constant is lacking in the picture (see Sect. 5.1). From this point of view, the $q$-quantization is more superficial than the $h$-quantization.

We end this Part by the extension of two classical algebraic properties [4]. A ring which has no zero divisor $\neq 0$ is called a domain: Noetherian means left and right Noetherian.

2.9.4. Theorem. Let $\mathbf{U}$ be an invertible q-enveloping algebra on $\mathbf{K}$. If $\mathbf{K}$ is a Noetherian domain, so is $\mathbf{U}$. In particular, in this case, $\mathbf{U}$ has a quotient division algebra (according to the Goldie theorem).

Proof. It suffices to prove the theorem for $\operatorname{gr}(\mathbf{U})$ [16], i.e. for $\mathbf{U}$ quadratic. We reason by induction on the number $p$ of generators of $\mathbf{U}$. If $p=1, \mathbf{U}=\mathbf{K}\left[e_{1}\right]$ and the result is classical. Suppose that the property holds for the $q$-enveloping algebras having $p-1$ generators. Let $\mathbf{U}$ be with generators $e_{1}, \ldots, e_{p}$. Consider $\mathbf{U}^{\prime}$ the subalgebra of $\mathbf{U}$ generated by $e_{1}, \ldots, e_{p-1}$. It is clear that $\mathbf{U}^{\prime}$ is a quadratic $q$-algebra. Noting that the generators defining $\mathscr{E}_{1}$ and $\mathscr{E}_{2}$ (Sect. 2.5) are linearly independent, we see that $\mathbf{U}^{\prime}$ is enveloping. Hence, $\mathbf{U}^{\prime}$ is a Noetherian domain. On the other hand, the $q$-PBW theorem shows that any element $a \in \mathbf{U}$ is uniquely written $a=\sum_{j} \alpha_{j} e_{p}^{j}, a_{j} \in \mathbf{U}^{\prime}$. Moreover, we have the commutation relations $e_{p} e_{i}=q_{p i} e_{i} e_{p}$ $+\left\langle e_{p}, e_{i}\right\rangle$. Then $\mathbf{U}$ is a skew polynomial algebra on $\mathbf{U}^{\prime}$ [16]. Its endomorphism $\sigma$ and its $\sigma$-derivation $\delta$ are defined by:

$$
\sigma\left(e_{i}\right)=q_{p i} e_{i} \text { and } \delta\left(e_{i}\right)=\left\langle e_{p}, e_{i}\right\rangle, \quad i<p .
$$

As the $q_{p i}$ are invertible, $\sigma$ is an automorphism. Theorem 1.2.9 of [16] allows to conclude that $\mathbf{U}$ is a Noetherian domain.

Among the non-classical examples of Sect. 2.7, we choose the following:

2.9.5. Corollary. Suppose that $\mathbf{K}$ is a Noetherian domain and that $q$ is invertible in K. The Manin algebras $\mathbf{M}_{q}(2, \mathbf{K}), \mathbf{G L}_{q}(2, \mathbf{K}), \mathbf{S L}_{q}(2, \mathbf{K})$ are Noetherian domains.

Proof. The assertion for $\mathbf{M}_{q}(2, \mathbf{K})$ obviously comes from the theorem and Example 2.7.6. Let us define $\mathbf{G} \mathbf{L}_{q}(2, \mathbf{K})$ in a different manner than Manin does (but 
the two versions are isomorphic). The quantum determinant [18] is $\mathbf{D}_{q}=a d-q^{-1} b c=$ $d s-q b c$. As $\mathbf{D}_{q}$ is central, the multiplicative monoid $\mathbf{S}$ generated by $\mathbf{D}_{q}$ is a regular Ore set. We set $\mathbf{G L}_{q}(2, \mathbf{K})=\mathbf{S}^{-1} \mathbf{M}_{q}(2, \mathbf{K})$ which is a Noetherian domain.

Now, fix $\lambda$ in $\mathbf{K}$. The algebra $\mathbf{A}_{\lambda}=\mathbf{M}_{q}(2, \mathbf{K}) /\left(\mathbf{D}_{q}-\lambda\right)$ is Noetherian again. The fact it is a domain is an easy consequence of the following lemma. By definition, $\mathbf{S L}_{q}(2)=\mathbf{A}_{1}$.

2.9.6. Lemma. The set of the monomials $a^{i} b^{j} d^{\ell}, a^{i} c^{k} d^{\ell}, i \geqq 0, j \geqq 0, k \geqq 1, \ell \geqq 0$ is $a$ basis of the K-module $\mathbf{A}_{\lambda}$.

Proof. Taking the associated graded algebra, we only examine the case $\lambda=0$. The $\mathbf{K}$-endomorphism $\rho$ of $\mathbf{M}_{q}(2, \mathbf{K})$ is defined by:

$$
\rho\left(a^{i} b^{j} c^{k} d^{\ell}\right)=q^{m(j+k-m)} a^{i+m} b^{j-m} c^{k-m} d^{\ell-m},
$$

where $m=\min (j, k)$. For all $x \in \mathbf{M}_{q}(2), x-\rho(x) \in\left(\mathbf{D}_{q}\right)$. We deduce $\operatorname{Ker} \rho=\left(\mathbf{D}_{q}\right)$. As $\rho \circ \rho=\rho, \mathbf{M}_{q}(2)=\operatorname{Ker} \rho \oplus \operatorname{Im} \rho$. Hence, $\mathbf{A}_{0}$ identifies to the $\mathbf{K}$-module $\operatorname{Im} \rho$. Now, it is clear that the monomials of the lemma form a basis of $\operatorname{Im} \rho$.

Notice that in Lemma 2.9.6, we can replace the basis by the following: $a^{j} b^{i} c^{\ell}$, $d^{k} b^{i} c^{\ell}$. In the case of $\mathbf{S L}_{q}(2)$, it is exactly the Woronowicz basis [25]. Woronowicz finds it by using an infinite dimensional representation by raising and lowering operators.

\section{The QPBW Theorem}

In all the sequel, the underlying ring is $\mathbf{K}=\mathbf{K}_{0}[[h]], \mathbf{K}_{0}$ a commutative ring and $h$ an inderminate (the Planck constant). The classical limit $h \rightarrow 0$ will be seen in a formal sense, i.e. relatively to the adic topology. This formal point of view, chosen by Drinfeld, has its source in the deformation quantization of Flato-Lichnérowicz [1].

3.1. The h-Adic Completion of a q-Enveloping Algebra. As in the previous part, $\mathbf{V}$ is a $\mathbf{K}$-module with a basis $\left(e_{1}, \ldots, e_{p}\right)$. We consider the tensor algebra $\mathbf{T}_{K}(\mathbf{V})$ in the $h$-adic topology. Its completion $\widehat{\mathbf{T}_{\boldsymbol{K}}(\mathbf{V})}$ is identified to $\mathbf{T}_{\boldsymbol{K}_{0}}(\mathbf{V})[[h]]$. So, any element $x$ in $\widehat{\mathbf{T}_{K}(\mathbf{V})}$ is uniquely written $x=\sum_{\ell \geqq 0} x_{\ell} h^{\ell}, x_{\ell} \in \mathbf{T}_{K_{0}}(\mathbf{V})$. Moreover, $x$ belongs to $\mathbf{T}_{K}(\mathbf{V})$ if and only if the degree of $x_{\ell}\left(\right.$ for the natural grading of $\mathbf{T}_{K_{0}}(\mathbf{V})$ ) is bounded when $\ell$ runs over $\mathbb{N}$. Let $\mathbf{U}$ be a $q$-algebra (Sect. 2.1 ). Its $h$-adic completion $\hat{\mathbf{U}}$ is

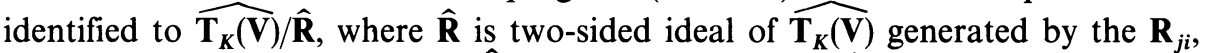
$i<j$. Hence, any element $a$ in $\hat{\mathbf{U}}$ is written $a=\sum_{\ell \geqq 0} a_{\ell} h^{\ell}$, where $a_{\ell}$ is the class modulo $\hat{\mathbf{R}}$ of an element $x_{\ell}$ of $\mathbf{T}_{K_{0}}(\mathbf{V})$. The element $a_{\ell}$ is a non-commutative polynomial with coefficients in $\mathbf{K}_{0}$. Precisely, if $\rho=\left(i_{1}, \ldots, i_{n}\right)$ is a sequence of indices in $\mathbf{I}=\{1, \ldots, p\}$, we denote by $\overline{e_{\rho}}$ the monomial $\overline{e_{i_{1}}} \ldots \overline{e_{i_{n}}}$ in $\mathbf{U}$ and we set $\overline{e_{\phi}}=1$. Then, $a_{\ell}=\sum_{\rho} c_{\rho \ell} \overline{e_{\rho}}$ (finite sum) with $c_{\rho \ell}$ in $\mathbf{K}_{0}$. It is clear that $a$ belongs to $\mathbf{U}$ if and only if $\operatorname{deg}\left(a_{\ell}\right)$ is bounded. Obviously the coefficients $a_{\ell}$ or $c_{\rho \ell}$ are not uniquely determined by $a$. However, if we notice that each $a_{\ell}$ is in $\mathbf{U}$, Theorem 2.3 .1 shows that we can reorder all the monomials $\overline{e_{\rho}}$ which appear in the series $\sum_{\ell \geqq 0} a_{\ell} h^{\ell}$. For 
conveniently formulating the latter statement and the QPBW theorem, we introduce the following terminology.

3.1.1. Definition. Let $a$ be an element in the $h$-adic completion $\hat{\mathbf{U}}$ of a q-algebra. An expansion of a such that:

$$
\begin{aligned}
a & =\sum_{\ell \geqq 0} a_{\ell} h^{\ell} \\
a_{\ell} & =\sum_{\boldsymbol{\rho}} c_{\rho \ell} \overline{e_{\rho}}, \quad c_{\rho \ell} \in \mathbf{K}_{0},
\end{aligned}
$$

where the sums (3.2) are finite, is called a quantum expansion of a. If all the monomials $\overline{e_{\rho}}$ in each $a_{\ell}$ are ordered (i.e. $\rho=\left(i_{1}, \ldots, i_{n}\right)$ with $i_{1} \leqq \cdots \leqq i_{n}$, or $\rho=\varnothing$ ), such $a$ quantum expansion is called ordered.

3.1.2. Theorem. Let $\mathbf{U}$ be a q-algebra on $\mathbf{K}=\mathbf{K}_{0}[[h]]$. Any element of the $h$-adic completion $\hat{\mathbf{U}}$ of $\mathbf{U}$ has an ordered quantum expansion. If, moreover, $\mathbf{U}$ is enveloping, this expansion is unique.

We have already said that the first assertion is a straightforward consequence of Theorem 2.3.1. The second assertion lies on the unicity of the $q$-PBW theorem, but also on the concept of semi-classical approximation which is quite common in Quantum Physics. This concept will have a precise meaning in our context. For each $r \in \mathbb{N}$, we set $\mathbf{K}(r)=\mathbf{K} /\left(h^{r+1}\right)$ and $\mathbf{U}(r)=\mathbf{U} /\left(h^{r+1}\right)=\hat{\mathbf{U}} /\left(h^{r+1}\right)$. The algebra $\mathbf{U}(r)$ is easily identified to $\mathbf{T}_{K(r)}(\mathbf{V}) / \mathbf{R}(r)$, where $\mathbf{R}(r)$ is the two-sided ideal of $\mathbf{T}_{K(r)}(\mathbf{V})$ generated by the $\mathbf{R}_{j i}$ modulo $h^{r+1}$. Then, it is clear that $\mathbf{U}(r)$ is a q-algebra on $\mathbf{K}(r)$ which is enveloping if $\mathbf{U}$ is. Naturally, $\hat{\mathbf{U}}$ is the projective limit of the $\mathbf{U}(r), r \in \mathbb{N}$.

3.1.3. Definition. The q-algebra $\mathbf{U}(0)$ is the classical approximation of the algebras $\mathbf{U}$ and $\hat{\mathbf{U}}$. The natural projections $\hat{\mathscr{L}}_{0}: \hat{\mathbf{U}} \rightarrow \mathbf{U}(0)$ and $\mathscr{L}_{0}=\left.\hat{\mathscr{L}}_{0}\right|_{\mathrm{U}}$ are the classical limits. For $r \geqq 1$, the q-algebra $\mathbf{U}(r)$ is the r-degree semi-classical approximation of $\mathbf{U}$ and $\hat{\mathbf{U}}$. The natural projections $\hat{\mathscr{L}}_{r}: \hat{\mathbf{U}} \rightarrow \mathbf{U}(r)$ and $\mathscr{L}_{r}=\left.\hat{\mathscr{L}}_{r}\right|_{U}$ are the r-degree semi-classical limits. On the contrary, we say that $\mathbf{U}($ or $\hat{\mathbf{U}})$ is a quantization of $\mathbf{U}(0)$.

This definition of quantization is quite compatible with Drinfeld's one [6].

Proof of Theorem 3.1.2 (unicity). Let us give an ordered quantum expansion of $a$ by the equalities (3.1) and (3.2). We omit the bar because $\mathbf{U}$ is enveloping. Then, $\hat{\mathscr{L}}_{r}(a)=\sum_{l=0}^{r}\left(\sum_{\rho} c_{\rho \ell} e_{\rho}\right) h^{\ell}$. In this formula, $e_{\rho}$ has to be read in $\mathbf{U}(r)$. Now, we can permute the two finite sums:

$$
\hat{\mathscr{L}}_{r}(a)=\sum_{\rho}\left(\sum_{\ell=0}^{r} c_{\rho \ell} h^{\ell}\right) e_{\rho} .
$$

Suppose $a=0$. Then, $\hat{\mathscr{L}}_{r}(a)=0$. The $q$-PBW for $\mathbf{U}(r)$ shows that $\sum_{\ell=0}^{r} c_{\rho \ell} h^{\ell}=0$ $(\operatorname{read}$ in $\mathbf{K}(r))$ for all $\rho$. Hence, all the $c_{\rho \ell}, 0 \leqq \ell \leqq r$, vanish. As $r$ is arbitrary, we get the result.

3.1.4. Remark. The previous proof indicates that $\operatorname{Ker} \hat{\mathscr{L}}_{r}$ is exactly the set of the ordered quantum series $\sum_{\ell \geqq 0} a_{\ell} h^{\ell}$ such that $a_{\ell}=0,0 \leqq \ell \leqq r$. 
3.2. The Obstruction $\mathrm{CPBW} \rightarrow \mathrm{QPBW}$. An easy consequence of the QPBW Theorem 3.1.2 is the following.

3.2.1. Theorem. If $\mathbf{U}$ is a q-enveloping algebra on $\mathbf{K}=\mathbf{K}_{0}[[h]]$, then $h$ is not a zero divisor in $\hat{\mathbf{U}}$.

Proof. Suppose $h a=0$, where the quantum expansion $a=\sum_{\ell \geqq 0} a_{\ell} h^{\ell}$ is ordered. Then, the quantum expansion $\sum_{\ell \geqq 0} a_{\ell} h^{\ell+1}$ is ordered and vanishes. Hence, all the
$a_{\ell}$ vanish and $a=0$.

We are going to locate the obstruction CPBW $\rightarrow$ QPBW. We only suppose that $\mathbf{U}$ is a $q$-algebra and that $\mathbf{U}(0)$ is an usual enveloping algebra on $\mathbf{K}_{0}$. If $a=\sum_{\ell \geqq 0} a_{\ell} h^{\ell}$ is an ordered quantum expansion in $\hat{\mathbf{U}}$ such that $a=0$, the CPBW theorem applied to $\hat{\mathscr{L}}_{0}(a)$ shows that $a_{0}=0$ (as in Theorem 3.1.2). We deduce $h a_{1}+h^{2} a_{2}+\cdots=0$. Now, we cannot a priori simplify by $h$ and carry on the process. Roughly speaking, we have

$$
\mathrm{QPBW}=\mathrm{CPBW}+\text { " } h \text { is not a zero divisor." }
$$

The essential role of our Jacobi condition (Definition 2.6.2) is to remove the obstruction CPBW $\rightarrow$ QPBW. It becomes clear with the following examples.

3.2.2. Example. We consider the algebra $\mathbf{U}$ generated by $e_{1}, e_{2}, e_{3}$, with the relations (1.1)-(1.3) of the Introduction. It is a scalar $q$-algebra. We find $J\left(e_{3}, e_{2}, e_{1}\right)=h^{2} e_{2}$. So, $\mathbf{U}$ is not enveloping. The Proposition 2.4.1 shows that $h^{2} \bar{e}_{2}=0$ in $\mathbf{U}$. But, it is obvious that $\bar{e}_{2} \neq 0$ in $\mathbf{U}$. Hence, $h$ is a zero divisor in $\mathbf{U}$ and there is no unicity of the ordered quantum series in $\hat{\mathbf{U}}$. We emphasize that $\mathbf{U}(0)$ is a usual enveloping algebra (it is the symmetric algebra).

3.3. Quantization of Two Classical Properties. We complete Theorem 2.9.4 in the following manner.

3.3.1. Theorem. Let $\mathbf{U}$ be a q-enveloping algebra as $\mathbf{K}=\mathbf{K}_{0}[[h]]$ and let $\mathbf{U}(0)$ be the classical approximation of $\mathbf{U}$. If $\mathbf{U}(0)$ is a Noetherian domain, so are $\mathbf{U}$ and $\hat{\mathbf{U}}$.

Proof. We simply paraphrase the proof of the well-known theorem: if $A$ is a domain (a Noetherian ring), so is $\mathbf{A}[[h]]$. This standard proof consists in associating to a non-vanishing series $\sum_{\ell \geqq 0} a_{\ell} h^{\ell}$ the first non-vanishing coefficient $a_{\ell}$, which allows to carry over the property from $\mathbf{A}$ to $\mathbf{A}[[h]][16]$. In case the series is an ordered quantum one, we have to associate $\mathscr{L}_{0}\left(a_{\ell}\right)$ which belongs to $\mathrm{U}(0)$. We leave the details to the reader.

3.3.2. Corollary. If $\mathbf{K}_{0}$ is a Noetherian domain and if $\mathbf{U}_{0}$ is a Sridharan enveloping algebra on $\mathbf{K}_{0}$, then any quantization (in the sense of Definition 3.1.3) of $\mathbf{U}_{0}$ is a Noetherian domain.

\section{The QPBW Theorem for $\mathscr{U}_{h}(s \ell(2))$}

4.1. Quantum Series in General. We need enlarge the framework of the Definition 3.1.1. The hypothesis $\mathbf{K}=\mathbf{K}_{0}[[h]]$ continues. Let $\mathbf{A}$ be an associative $\mathbf{K}$-algebra 
with unit which is complete for the h-adic topology. We fix an ordered family $\left(x_{1}, \ldots, x_{n}\right)$ elements in A. A series $a=\sum_{\ell \geqq 0} a_{\ell} h^{\ell}$ in $\mathbf{A}$ such that $a_{\ell}=\sum_{\alpha} c_{\alpha \ell} x_{1}^{\alpha_{1}} \cdots x_{n}^{\alpha_{n}}$ (finite sum on $\left.\alpha=\left(\alpha_{1}, \ldots, \alpha_{n}\right) \in \mathbb{N}^{n}\right), c_{\alpha \ell} \in \mathbf{K}_{0}$, is called an ordered quantum series relatively to the ordered family $\left(x_{1}, \ldots, x_{n}\right)$. The family $\left(x_{1}, \ldots, x_{n}\right) h$-generates $\mathbf{A}$ if any element of $\mathbf{A}$ has an ordered quantum expansion relative to this family. The family is $h$-free if an ordered quantum series which vanishes has all its coefficients $c_{\alpha \ell}=0$. If the family $h$-generates $\mathbf{A}$ and is $h$-free, we say that it is $a h$-basis of $\mathbf{A}$. In this case, the product in $\mathbf{A}$ is entirely determined by the ordered quantum expansions of the products $x_{j} x_{i}, 1 \leqq i<j \leqq n$. An example of $h$-basis is provided by the QPBW theorem (3.1.2).

4.2. The Algebra $\mathscr{U}_{h}(s \ell(2))$. From now on, $\mathbf{V}$ is a vector space on $\mathbf{K}_{0}=\mathbb{C}$ with a basis $\left(\mathbf{X}_{+}, \mathbf{X}_{-}, \mathbf{H}\right)$. Introduced by Kulish-Reshetikhin [13], $\mathscr{U}_{h}(\operatorname{s} \ell(2))$ is the reduction of $\mathbf{T}_{\mathbb{C}}(\mathbf{V})[[h]]$ modulo the ideal generated by the following elements:

$$
\begin{gathered}
\mathbf{H} \otimes \mathbf{X}_{ \pm}-\mathbf{X}_{ \pm} \otimes \mathbf{H} \mp 2 \mathbf{X}_{ \pm}, \\
\mathbf{X}_{+} \otimes \mathbf{X}_{-}-\mathbf{X}_{-} \otimes \mathbf{X}_{+}-\frac{2}{h} \operatorname{sh}\left(\frac{h}{2} \mathbf{H}\right) .
\end{gathered}
$$

The complete K-algebra $\mathscr{U}_{h}(s \ell(2))$ is a quantization (in the Drinfeld sense) of the usual enveloping algebra $\mathscr{U}(s \ell(2))$ since we have $\mathscr{U}_{h}(s \ell(2)) /(h)=\mathscr{U}(s \ell(2))$.

4.2.1. Theorem. With the above notations, the family $\left(\mathbf{X}_{+}, \mathbf{X}_{-}, \mathbf{H}\right)$ is a $h$-basis of $\mathscr{U}_{h}(s \ell(2))$.

The relation (4.2) is not quadratic. Hence, we cannot consider $\mathscr{U}_{h}(s \ell(2))$ as the completion of a $q$-enveloping algebra. For proving Theorem 4.2.1, we first linearize the bracket $\left[\mathbf{X}_{+}, \mathbf{X}_{-}\right]$, i.e. we "replace" $\mathbf{H}$ by the two generators $\left.\frac{1}{h} \exp \left( \pm \frac{h}{2} \mathbf{H}\right)-1\right)$, which essentially amounts to take Jimbo's generators [11]. Some $q$-commutators are arising from this linearization and the Jacobi condition may be used (see the following section). Then, Theorem 4.2.1 is easily derived from the fact that the linearization is a trivial extension of $\mathscr{U}_{h}(s \ell(2))$.

4.3. Linearization of $\mathscr{U}_{h}(s \ell(2))$. We introduce the $\mathbf{K}$-algebra $\mathbf{U}$ generated by $\mathbf{X}_{+}, \mathbf{X}_{-}, f_{+}, f_{-}$with the following relations:

$$
\begin{aligned}
\mathbf{X}_{+} \mathbf{X}_{-}-\mathbf{X}_{-} \mathbf{X}_{+} & =f_{+}-f_{-}, \\
f_{+} f_{-} & =f_{-} f_{+}, \\
f_{+} \mathbf{X}_{ \pm}-e^{ \pm h} \mathbf{X}_{ \pm} f_{+} & =\frac{1}{h}\left(e^{ \pm h}-1\right) \mathbf{X}_{ \pm}, \\
f_{-} \mathbf{X}_{ \pm}-e^{\mp h} \mathbf{X}_{ \pm} f_{-} & =\frac{1}{h}\left(e^{\mp h}-1\right) \mathbf{X}_{ \pm} .
\end{aligned}
$$

4.3.1. Theorem. The $\mathbf{K}$-algebra $\mathbf{U}$ is a q-enveloping algebra.

Proof. Obviously, $\mathbf{U}$ is a linear and invertible $q$-algebra. We compute the $q$-Jacobi sums for the ordering $\mathbf{X}_{+}<\mathbf{X}_{-}<f_{+}<f_{-}$. In fact, the involution $\mathbf{X}_{+} \leftrightarrow \mathbf{X}_{-}, f_{+} \leftrightarrow f_{-}$. 
allows to confine us to $\mathbf{J}\left(f_{+}, \mathbf{X}_{-}, \mathbf{X}_{+}\right)$and $\mathbf{J}\left(f_{-}, f_{+}, \mathbf{X}_{+}\right)$. We get:

$$
\begin{aligned}
\mathbf{J}\left(f_{+}, \mathbf{X}_{-}, \mathbf{X}_{+}\right)= & f_{-} \otimes f_{+}-f_{+} \otimes f_{-}, \\
\mathbf{J}\left(f_{-}, f_{+}, \mathbf{X}_{+}\right)= & \frac{1}{h}\left(e^{-h}-1\right)\left[f_{+} \otimes \mathbf{X}_{+}-e^{h} \mathbf{X}_{+} \otimes f_{+}-\frac{1}{h}\left(e^{h}-1\right) \mathbf{X}_{+}\right] \\
& -\frac{1}{h}\left(e^{h}-1\right)\left[f_{-} \otimes \mathbf{X}_{+}-e^{-h} \mathbf{X}_{+} \otimes f_{-}-\frac{1}{h}\left(e^{-h}-1\right) \mathbf{X}_{+}\right] .
\end{aligned}
$$

These two sums belong to the submodule $\mathscr{E}_{1}$, hence the Jacobi condition holds.

The QPBW theorem (3.1.2) shows that the family $\left(\mathbf{X}_{+}, \mathbf{X}_{-}, f_{+}, f_{-}\right)$is a $h$-basis of U. Wet set:

$$
g_{ \pm}=\frac{2}{h} \log \left(1+h f_{ \pm}\right)
$$

The right-hand side of (4.9) is a quantum series with $2 f_{ \pm}$as first term. Hence, $\left(\mathbf{X}_{+}, \mathbf{X}_{-}, g_{+}, g_{-}\right)$is also $a h$-basis of $\hat{\mathbf{U}}$. Relatively to this $h$-basis, the product of $\hat{\mathbf{U}}$ is entirely determined by the following brackets:

$$
\begin{aligned}
{\left[g_{+}, \mathbf{X}_{ \pm}\right] } & = \pm 2 \mathbf{X}_{ \pm}, \\
{\left[g_{-}, \mathbf{X}_{ \pm}\right] } & =\mp 2 \mathbf{X}_{ \pm}, \\
{\left[g_{+}, g_{-}\right] } & =0 \\
{\left[\mathbf{X}_{+}, \mathbf{X}_{-}\right] } & =\frac{1}{h}\left(\exp \left(\frac{h}{2} g_{+}\right)-\exp \left(\frac{h}{2} g_{-}\right)\right) .
\end{aligned}
$$

Comparing with (4.1) and (4.2), we see that $\varphi\left(\mathbf{X}_{ \pm}\right)=\mathbf{X}_{ \pm}, \varphi\left(g_{ \pm}\right)= \pm \mathbf{H}$ define an algebra morphism $\varphi: \hat{\mathbf{U}} \rightarrow \mathscr{U}_{h}(s \ell(2))$. The element $u=\frac{1}{2}\left(g_{+}+g_{-}\right)$belongs to $\operatorname{Ker} \varphi$ and the morphism $\bar{\varphi}: \hat{\mathbf{U}} /(u) \rightarrow \mathscr{U}_{h}(s \ell(2))$ obtained by reduction is characterized by:

$$
\begin{aligned}
\bar{\varphi}\left(\overline{\mathbf{X}_{ \pm}}\right) & =\mathbf{X}_{ \pm}, \\
\bar{\varphi}\left(\overline{g_{+}}\right) & =\mathbf{H} .
\end{aligned}
$$
Now, we introduce the following elements in $\hat{\mathbf{U}}: \mathbf{X}_{ \pm}^{\prime}=\mathbf{X}_{ \pm} \exp \left(-\frac{h u}{4}\right)$. As $u$ is
central in $\hat{\mathbf{U}}$, we easily deduce from (4.10)-(4.13),

$$
\begin{aligned}
{\left.\left[\frac{1}{2}\left(g_{+}-g_{-}\right), \mathbf{X}_{ \pm}^{\prime}\right)\right] } & = \pm 2 \mathbf{X}_{ \pm}^{\prime}, \\
{\left[\mathbf{X}_{+}^{\prime}, \mathbf{X}_{-}^{\prime}\right] } & =\frac{2}{h} \operatorname{sh}\left(\frac{h}{4}\left(g_{+}-g_{-}\right)\right) .
\end{aligned}
$$

Hence, $\sigma\left(\mathbf{X}_{ \pm}\right)=\mathbf{X}_{+}^{\prime}, \sigma(\mathbf{H})=\frac{1}{2}\left(g_{+}-g_{-}\right)$define an algebra morphism $\sigma: \mathscr{U}_{h}(s \ell(2)) \rightarrow \hat{\mathbf{U}}$ such that $\varphi^{\circ} \sigma=\mathrm{I} \overline{\mathrm{d}}$, and we obtain:

4.3.2. Theorem. The morphism $\bar{\varphi}: \hat{\mathbf{U}} /(u) \rightarrow \mathscr{U}_{h}(s \ell(2))$ defined by (4.14)-(4.15) is an isomorphism. Moreover, the extension of algebras

$$
0 \rightarrow(u) \rightarrow \hat{\mathbf{U}} \stackrel{\varphi}{\longrightarrow} \mathscr{U}_{h}(s \ell(2)) \rightarrow 0
$$

is trivial. That means there is a morphism $\sigma$ which is a section of $\varphi$. 
Proof of Theorem 4.2.1. The family $\left(\mathbf{X}_{+}, \mathbf{X}_{-}, g_{+}, g_{-}\right) h$-generates $\hat{\mathbf{U}}$. Then, its image $\left(\mathbf{X}_{+}, \mathbf{X}_{-}, \mathbf{H}\right)$ by $\varphi h$-generates $\mathscr{U}_{h}(s \ell(2))$. On the other hand, the family $\left(\mathbf{X}_{+}, \mathbf{X}_{-}, \frac{1}{2}\left(g_{+}-g_{-}\right)\right)$is clearly $h$-free in $\hat{\mathbf{U}}$. Now, the quantum series $\exp \left(-\frac{h u}{4}\right)$ is central in $\hat{\mathbf{U}}$ and has 1 as first term. Hence, $\left(\mathbf{X}_{+}^{\prime}, \mathbf{X}_{-}^{\prime}, \frac{1}{2}\left(g_{+}-g_{-}\right)\right)$is also $h$-free in $\hat{\mathbf{U}}$. But the latter family is the image under $\sigma$ of $\left(\mathbf{X}_{+}, \mathbf{X}_{-}, \mathbf{H}\right)$. We conclude that $\left(\mathbf{X}_{+}, \mathbf{X}_{-}, \mathbf{H}\right)$ is $h$-free in $\mathscr{U}_{h}(s \ell(2))$.

\section{The Quantum Filtration}

5.1. The Planck Constant Has Degree - 1. We have noticed (Corollary 2.9.3) that the $q$-enveloping algebras which are naturally filtered are never almost commutative except for the usual ones. For restoring the almost commutativity for a larger class of algebras, we adopt a new filtration, called the quantum filtration. As a matter of fact, we do not develop all the consequences of this choice in this paper. We just study the associated graded algebras, seen as classical objects. According to the works of Brylinski [3] and Kassel [12], an interesting application would be the computation of the homology of quantum algebras.

The terminology of Sect. 4.1 is used again. We suppose that the complete associative $\mathbf{K}$-algebra $\mathbf{A}$ has $a$ h-basis $\left(x_{1}, \ldots, x_{n}\right)$. Let $a$ be an element of $\mathbf{A}$. We write its ordered quantum expansion as $a=\sum_{\alpha \ell} c_{\alpha \ell} x_{1}^{\alpha_{1}} \cdots x_{n}^{\alpha_{n}} h^{\ell}$. We say that $a$ has degree $m(m \in \mathbb{Z})$ if the condition $c_{\alpha \ell} \neq 0$ implies $\alpha_{1}+\cdots+\alpha_{n}-\ell \leqq m$. The submodule of the elements with degree $m$ is denoted by $\mathbf{F}_{m}(\mathbf{A})$. Clearly, $\left(\mathbf{F}_{m}(\mathbf{A})\right)_{m \in \mathbb{Z}}$ is an increasing $\mathbb{Z}$-filtration of $\mathbf{A}$ such that: $\bigcap_{m \in \mathbb{Z}} \mathbf{F}_{m}(\mathbf{A})=\{0\}, \mathbf{F}_{m}(\mathbf{A}) \cdot \mathbf{F}_{n}(\mathbf{A}) \subseteq \mathbf{F}_{m+n}(\mathbf{A})$. The union $\mathbf{F}(\mathbf{A})$ of the $\mathbf{F}_{m}(\mathbf{A})$ is not $\mathbf{A}$, but is dense in $\mathbf{A}$. We denote by $\mathbf{G}(\mathbf{A})=\bigoplus_{m \in \mathbb{Z}} \mathbf{G}_{m}(\mathbf{A})$ the graded algebra associated to $\mathbf{F}(\mathbf{A})$ and by $\mathbf{C}(\mathbf{A})$ the completion of $\mathbf{G}(\mathbf{A})$ for the $h$-adic topology.

5.1.1. Definition. With the above notations, the algebra $\mathbf{A}$ endowed with the quantum filtration $\left(\mathbf{F}_{m}(\mathbf{A})\right)_{m \in \mathbb{Z}}$ is called a quantum object. The graded algebra $\mathbf{C}(\mathbf{A})$ is called the classical object associated to $\mathbf{A}$. The algebra $\mathbf{A}$ is said almost commutative if $\mathbf{C}(\mathbf{A})$ is commutative.

5.1.2. Theorem. Let $\mathbf{U}$ be a q-enveloping algebra on $\mathbf{K}=\mathbf{K}_{0}[[h]]$ (notations of Sect. 2.1). The completion $\hat{\mathbf{U}}$ is almost commutative if and only if, for all $i<j$, $q_{j i}=1(\bmod . h)$ and $\overline{\left\langle e_{j}, e_{i}\right\rangle}$ has degree 1 .

Proof. It is immediate from the following commutation relation:

$$
e_{j} e_{i}-e_{i} e_{j}=\left(q_{j i}-1\right) e_{i} e_{j}+\overline{\left\langle e_{j}, e_{i}\right\rangle} .
$$

The right-hand side of (5.1) (which has degree 2) will have degree 1 if and only if each term has degree 1 (because of $(\mathrm{S})$ ).

Practically, it is easy to verify the almost commutativity of $\hat{\mathbf{U}}$. For example, the Manin algebra $\mathbf{M}_{q}(2)$ is almost commutative when $q=e^{h / 2}$ (the Drinfeld parameter [6]). In this case, the quadratic part $\left(q-q^{-1}\right) b c$ of (2.29) (Example 2.7.6) has really 
degree 1 . Obviously, any affine $q$-enveloping algebra such that $q_{j i} \equiv 1(\bmod h), i<j$, is almost commutative.

An example of almost commutative algebra which is not the completion of a $q$-enveloping algebra is provided by $\mathscr{U}_{h}(\operatorname{sl}(2))$. Indeed, the brackets $\left[\mathbf{H}, \mathbf{X}_{ \pm}\right]$and $\left[\mathbf{X}_{+}, \mathbf{X}_{-}\right]$have degree 1 (see (4.1)-(4.2)). More generally, we conjecture that any Drinfeld-Jimbo quantum algebra $\mathscr{U}_{h}(\mathfrak{g})$, where $\mathfrak{g}$ is a Kac-Moody algebra, has a $h$-basis and is almost commutative for the quantum filtration.

We return to the general case of an almost commutative algebra $\mathbf{A}$. The description of the classical object $\mathbf{C}(\mathbf{A})$ is straightforward. It is clear that the algebra $\mathbf{C}(\mathbf{A})$ coincides with the commutative algebra $\mathbf{K}_{0}\left[x_{1}, \ldots, x_{n}\right][[h]]$. The grading of C(A) is such that $x_{1}, \ldots, x_{n}$ have degree 1 and $h$ has degree -1 . The subalgebra $\mathbf{G}(\mathbf{A})$ is the set of series in $h$ having a finite degree.

As usual, the commutative classical object $\mathbf{C}(\mathbf{A})$ is naturally a Poisson algebra (see e.g. Kassel [2]). Let us outline the Poisson bracket of two elements $\alpha$ and $\beta$ of $\mathbf{G}(\mathbf{A}), \operatorname{deg}(\alpha)=m, \operatorname{deg}(\beta)=n$. If $\alpha(\beta)$ is the class of $a \in \mathbf{F}_{m}(\mathbf{A})\left(b \in \mathbf{F}_{n}(\mathbf{A})\right)$, the commutator $a b-b a$ belongs to $\mathbf{F}_{m+n-1}(\mathbf{A})$ and its class in $\mathbf{G}_{m+n-1}(\mathbf{A})$ only depends on $\alpha$ and $\beta$. This class is the Poisson bracket $\{\alpha, \beta\}$ of $\alpha$ and $\beta$. Clearly, $\{$,$\} is$ extended to $\mathbf{C}(\mathbf{A})$ by density. So, $\mathbf{C}(\mathbf{A})$ is a graded Poisson algebra.

5.1.3. Example. The Poisson bracket of the classical object $\mathbf{C}\left(\mathbf{M}_{q}(2)\right)$ is defined on the generators $a, b, c, d$ as follows. Any bracket of two distinct generators vanishes except $\{d, a\}=-\{a, d\}=h b \times c$.

5.1.4. Example. The Poisson bracket of the classical object $\mathbf{C}\left(\mathscr{U}_{h}(s \ell(2))\right.$ is defined on the generators $\mathbf{X}_{+}, \mathbf{X}_{-}, \mathbf{H}$ by the following formulas:

$$
\begin{gathered}
\left\{\mathbf{H}, \mathbf{X}_{ \pm}\right\}= \pm 2 \mathbf{X}_{ \pm}, \\
\left\{\mathbf{X}_{+}, \mathbf{X}_{-}\right\}=\frac{2}{h} \operatorname{sh}\left(\frac{h}{2} \mathbf{H}\right) .
\end{gathered}
$$

5.1.5. Remark. The presence of the Planck constant $h$ in the description of the "classical" object $\mathbf{C}(\mathbf{A})$ seems strange. Actually, it is possible to have some bijective quantization correspondences (in our sense [2]) between $\mathbf{C}(\mathbf{A})$ and $\mathbf{A}$, which is impossible between $\mathbf{U}(0)$ and $\hat{\mathbf{U}}$ (because of the difference of dimension).

5.2. Filtered Coproducts. We keep the notations of 5.1. It is clear that the complete algebra $\mathbf{A} \hat{\otimes} \mathbf{A}$ has a $h$-basis. Then, we can endow it with the quantum filtration. As $\mathbf{A}$ is almost commutative, $\mathbf{A} \hat{\otimes} \mathbf{A}$ is also almost commutative. Let us give a coproduct $\Delta$ on $\mathbf{A}$, i.e. a continuous $\mathbf{K}$-linear mapping $\Delta: \mathbf{A} \rightarrow \mathbf{A} \hat{\otimes} \mathbf{A}$. We suppose $\Delta$ to be coassociative and compatible with the product of $\mathbf{A}$ (i.e. $\Delta$ is an algebra morphism). So, $\Delta$ is entirely determined by the data of the $\Delta\left(x_{i}\right), 1 \leqq i \leqq n$. The Hopf algebra $\hat{\mathbf{U}}$ becomes filtered if we suppose that $\Delta$ is filtered, i.e. each $\Delta\left(x_{i}\right)$ has degree 1 . With all these hypotheses, the graded linear mapping $\mathbf{C}(\Delta)$ associated to $\Delta$ makes $\mathbf{C}(\mathbf{A})$ a graded Hopf algebra. As $\mathbf{C}(\Delta)$ is a Poisson morphism, we conclude that $\mathbf{C}(\mathbf{A})$ is a graded Poisson-Hopf algebra (in the Drinfeld sense [5]). All the previous hypotheses hold for the coproduct of $\mathscr{U}_{h}(\operatorname{sl}(2))$ (see its definition in [6]). Here again, these hypotheses should be true for the coproduct of a general Drinfeld-Jimbo quantum algebra $\mathscr{U}_{h}(\mathfrak{g})$. 
Acknowledgements. The author would like to thank Prof. T. Fack for his interest in this work and his encouragement during its realization. He also wants to express his thanks to M. Rosso for valuable discussions and suggestions.

\section{References}

1. Bayen, F., Flato, M., Fronsdal, C., Lichnérowicz, A., Sternheimer, D.: Deformation theory and quantization. Ann. Phys. 111, 61-151 (1978)

2. Berger, R.: Algebraic quantization. Lett. Math. Phys. 17, 275-283 (1989)

3. Brylinski, J. L.: A differential complex for Poisson manifolds. J. Diff. Geom. 28, 93-114 (1988)

4. Dixmier, J.: Algèbres enveloppantes. Paris: Gauthier-Villars 1974

5. Drinfel'd, V. G.: Hopf algebras and the quantum Yang-Baxter equation. Soviet. Math. Dokl. 32, 254-258 (1985)

6. Drinfel'd, V. G.: Quantum groups. In Proc. ICM, Berkeley, 1986. Providence, RI: Am. Math. Soc. 1988

7. Hayashi, T.: $Q$-analogues of Clifford and Weyl algebras-Spinor and oscillator representations of quantum enveloping algebras. Commun. Math. Phys. 127, 129-144 (1990)

8. Jacobson, N.: Lie algebras. New York: Wiley 1962

9. Jannussis, A., Brodimas, G., Sourlas, D.: Remarks on the q-quantization. Lett. Nuovo Cimento 30, 123-127 (1981)

10. Jategaonkar, V. A.: A multiplicative analogue of the Weyl algebra. Commun. Algebra 12, $1669-1688$ (1984)

11. Jimbo, M.: A $q$-difference analogue of $\mathbf{U}(\mathbf{g})$ and the Yang-Baxter equation. Lett. Math. Phys. 10, 63-69 (1985)

12. Kassel, C.: L'homologie cyclique des algèbres enveloppantes. Invent. Math. 91, 221-251 (1988)

13. Kulish, P. P., Reshetikhin, N. Y.: The quantum linear problem for the sine-Gordon equation and higher representations. J. Soviet. Math. 23, 2435 (1983)

14. Kuryshkin, M. V.: Opérateurs quantiques généralisés de création et d'annihilation. Ann. Fond. L. de Broglie 5, 111-125 (1980)

15. Lusztig, G.: Quantum groups at roots of 1. Geom. Dedi. 35, 89-113 (1990)

16. Mc Connell, J. C., Robson, J. C.: Non-commutative Noetherian rings. New York: Wiley 1987

17. Manin, Y. I.: Some remarks on Koszul algebras and quantum groups. Ann. Inst. Fourier 38, 191-205 (1988)

18. Manin, Y. I.: Quantum groups and non-commutative geometry. Pub. Centre Rech. Math., University Montréal 1988

19. Noumi, M., Mimachi, K.: Quantum 2-spheres and big $q$-Jacobi polynomials. Commun. Math. Phys. 128, 521-531 (1990)

20. Podles, P.: Quantum spheres. Lett. Math. Phys. 14, 193-202 (1987)

21. Priddy, S. B.: Koszul resolutions, Trans. A.M.S. 152, 39-60 (1970)

22. Rosso, M.: Groupes quantiques, représentations linéaires et applications. Thèse University Paris VII 1990

23. Sridharan, R.: Filtered algebras and representations of Lie algebras. Trans. Am. Math. Soc. 100, 530-550 (1961)

24. Wallisser, R.: Rationale approximation der $q$-Analogous der Exponentialfunktion und Irrationalitätsaussagen für diese Funktion. Arch. Math. 44, 59-64 (1985)

25. Woronowicz, S. L.: Twisted SU(2) group. An example of a noncommutative differential calculus. Publ. RIMS 23, 117-181 (1987)

Communicated by A. Connes 